Pure and Applied Mathematics Quarterly

Volume 2, Number 3

(Special Issue: In honor of

Robert MacPherson, Part 1 of 3)

$893-914,2006$

\title{
Hypersurface Singularities and Milnor Equisingularity
}

\author{
Lê Dũng Tráng and David B. Massey \\ In honor of Robert MacPherson on his 60th birthday
}

\section{INTRODUCTION}

Let $\mathcal{U}$ be an open neighborhood of the origin in $\mathbb{C}^{n+1}$, let $f:(\mathcal{U}, \mathbf{0}) \rightarrow(\mathbb{C}, 0)$ be complex analytic, and let $s$ denote the dimension of the critical locus $\Sigma f$ at the origin.

We will use $\mathbf{x}:=\left(x_{0}, \ldots, x_{n}\right)$ to denote the standard coordinate functions on $\mathbb{C}^{n+1}$. We will use $\mathbf{z}:=\left(z_{0}, \ldots, z_{n}\right)$ to denote arbitrary analytic local coordinates on $\mathcal{U}$ near the origin. All of our constructions and results will depend only on the linear part of the coordinates $\mathbf{z}$; hence, when we say that the $\mathbf{z}$ are chosen generically, we mean that the linear part of $\mathbf{z}$ consists of a generic linear combination of $\mathbf{x}$ (generic in $\operatorname{PGL}\left(\mathbb{C}^{n+1}\right)$ ).

Let $F_{f}=F_{f, \mathbf{0}}$ denote the Milnor fiber of $f$ at the origin. It is well-known (see [9]) that the reduced integral homology, $\widetilde{H}_{k}\left(F_{f}\right)$, of $F_{f}$ can be non-zero only for $n-s \leq k \leq n$, and is free Abelian in degree $n$. Cohomologically, this means that $\widetilde{H}^{k}\left(F_{f}\right)$ can be non-zero only for $n-s \leq k \leq n$, and is free Abelian in degree $n-s$. For a general reference to non-isolated hypersurface singularities, see [26].

For $s>0$ and arbitrary $f$, it is not known how to calculate, algebraically, the groups $\widetilde{H}_{*}\left(F_{f}\right)$ or their ranks. Even for $s=1$, there is no effective, general method for calculating the ranks of $\widetilde{H}_{n-1}\left(F_{f}\right)$ and $\widetilde{H}_{n}\left(F_{f}\right)$. However, there are a number of known bounds on the Betti numbers of $F_{f}$; we need to describe one of these bounds.

Received November 4, 2005.

The second author would like to thank the Abdus Salam ICTP for their hospitality; most of this paper was written during a visit there.

AMS subject classifications: 32B15, 32C35, 32C18, 32B10.

Keywords: hypersurface singularity, Milnor fiber, swing, polar curve, vanishing cycles, discriminant, Cerf diagram, intersection diagram, perverse sheaves. 
For each $s$-dimensional component, $\nu$, of $\Sigma f$, for a generic point $p \in \nu$, for a generic codimension $s$ (in $\mathcal{U}$ ) affine linear subspace, $N$, (a normal slice) containing $p$, the function $f_{\left.\right|_{N}}$ has an isolated critical point at $p$ and the Milnor number at $p$ is independent of the choices; we let $\stackrel{\circ}{\mu}_{\nu}$ denote this common value.

Let $V\left(z_{0}, \ldots, z_{s-1}\right)$ denote the zero locus of the coordinate functions $z_{0}, \ldots, z_{s-1}$. If the coordinates $\left(z_{0}, \ldots, z_{s-1}\right)$ are such that $f_{\left.\right|_{V\left(z_{0}, \ldots, z_{s-1}\right)}}$ has an isolated critical point at the origin, then the $s$-dimensional Lê number [17], $\lambda_{f, \mathbf{z}}^{s}(\mathbf{0})$, at the origin is defined, and $\lambda_{f, \mathbf{z}}^{s}(\mathbf{0})$ is a sum of intersection numbers: $\lambda_{f, \mathbf{z}}^{s}(\mathbf{0})=$ $\sum_{\nu} \stackrel{\circ}{\mu}_{\nu}\left(\nu \cdot V\left(z_{0}, \ldots, z_{s-1}\right)\right)_{0}$, where the sum is over the $s$-dimensional (reduced) components $\nu$ of $\Sigma f$. If the coordinates $\left(z_{0}, \ldots, z_{s-1}\right)$ are sufficiently generic, then $\lambda_{f, \mathbf{z}}^{s}(\mathbf{0})$ obtains its minimum value of $\sum_{\nu} \stackrel{\circ}{\mu}_{\nu}$ mult $_{\mathbf{0}} \nu$; we denote this generic value by $\lambda_{f}^{s}(\mathbf{0})$ (with no subscript by the coordinates). Theorem 3.3 of [17] implies that $\tilde{b}_{n-s}:=\operatorname{rank} \widetilde{H}_{n-s}\left(F_{f}\right)=\operatorname{rank} \widetilde{H}^{n-s}\left(F_{f}\right) \leq \lambda_{f}^{s}(\mathbf{0})$.

We wish to consider families of singularities. Fix a set of local coordinates $\mathbf{z}$ for $\mathcal{U}$ at the origin. Let $G:=\left(z_{0}, \ldots, z_{s-1}\right)$. If $\mathbf{q} \in \mathcal{U}$, we define $f_{\mathbf{q}}:=f_{\left.\right|_{G^{-1}(G(\mathbf{q}))}}$.

Definition 1.1.. We say that $f_{\mathbf{q}}$ is a simple $\mu$-constant family at the origin if and only if, at the origin, $f_{\mathbf{0}}$ has an isolated critical point, $\Sigma f$ is smooth, $G_{\left.\right|_{\Sigma f}}$ is a submersion and, for all $\mathbf{q} \in \Sigma f$ close to the origin, the Milnor number $\mu_{\mathbf{q}}\left(f_{\mathbf{q}}\right)$ is independent of $\mathbf{q}$.

Our interest in simple $\mu$-constant families stems from the fact that they have many "equisingularity" properties; see Theorem 2.3. In particular, if $n-s \neq 2$ and $f_{\mathbf{q}}$ is a simple $\mu$-constant family at the origin, then the main theorem of Lê and Ramanujam in [14] implies that the local, ambient, topological-type of $V\left(f_{\mathbf{q}}\right)$ at $\mathbf{q}$ is independent of the point $\mathbf{q} \in \Sigma f$ near the origin.

We can now state our main theorem, which tells us that the rank of $\widetilde{H}_{n-s}\left(F_{f}\right)$ completely determines whether or not $f$ defines a simple $\mu$-constant family.

Main Theorem (Theorem 5.3). Suppose that $\operatorname{dim}_{\mathbf{0}} \Sigma\left(f_{\mathbf{0}}\right)=0$.

Then, the rank, $\tilde{b}_{n-s}$, of $\widetilde{H}_{n-s}\left(F_{f}\right)$ is equal to $\lambda_{f, \mathbf{z}}^{s}(\mathbf{0})$ if and only if $f_{\mathbf{q}}$ is a simple $\mu$-constant family.

This general case of the Main Theorem actually follows quickly from the 1dimensional case;

Theorem (Theorem 5.1). Suppose that $\operatorname{dim}_{\mathbf{0}} \Sigma f=1$, and $\operatorname{dim}_{\mathbf{0}} \Sigma\left(f_{\left.\right|_{V\left(z_{0}\right)}}\right)=0$. Then, the following are equivalent: 
a) $f_{\mathbf{q}}:=f_{\left.\right|_{0} ^{-1}\left(z_{0}(\mathbf{q})\right)}$ is a simple $\mu$-constant family;

b) $\operatorname{rank} \widetilde{H}_{n-1}\left(F_{f}\right)=\lambda_{f, z_{0}}^{1}(\mathbf{0})$;

c) there exists a prime $p$ such that $\operatorname{dim} \widetilde{H}_{n-1}\left(F_{f} ; \mathbb{Z} / p \mathbb{Z}\right)=\lambda_{f, z_{0}}^{1}(\mathbf{0})$.

Thus, if we are not in the simple $\mu$-constant case, $\operatorname{rank} \widetilde{H}_{n-1}\left(F_{f}\right)<\lambda_{f, z_{0}}^{1}(\mathbf{0})=$ $\lambda_{f}^{1}(\mathbf{0})$, and this inequality holds with $\mathbb{Z} / p \mathbb{Z}$ coefficients.

In [30], M. Tibăr investigates, on the homotopy level, the Milnor fiber of hypersurfaces with one-dimensional singular sets. Corollary 4.2 of that paper provides an independent proof of our Theorem 5.1.

The remaining sections of this paper are organized as follows. In Section 2, we prove that many different notions of "Milnor equisingularity" are all equivalent. In Section 3, we recall a number of known results on the topology of the Milnor fiber. In Section 4, we provide a careful construction of the "swing homotopy", which is essential for the proof of the Main Theorem. Section 5 contains the proof of the Main Theorem. As a corollary to our Main Theorem, we show that it implies that the vanishing cycles of $f$, as an object in the category of perverse sheaves, cannot be semi-simple in non-trivial cases. In the final section of this paper, Section 6, we make some final remarks and present counterexamples to some conceivable "improvements" to the statement of the Main Theorem.

\section{Milnor Equisingularity}

There are many conceivable definitions of what one might wish to call a "simple" $\mu$-constant family. The definition that we use in Definition 1.1 may seem too strong; we used this strong characterization so that it would be clear in the Main Theorem that the condition $\tilde{b}_{n-s}=\lambda_{f, \mathbf{z}}^{s}(\mathbf{0})$ implies that we are in a very trivial case.

In this section, we will show that all other reasonable concepts of $\mu$-constant families are equivalent. In the case $s=1$, these equivalences are a combination of the results Lê and Saito in [15], Lê and Ramanujam in [14], Teissier in 1.2 of [28], and the non-splitting result proved independently by Gabrielov [8], Lazzeri [10], and Lê [12].

Suppose that $\operatorname{dim}_{\mathbf{0}} \Sigma\left(f_{\mathbf{0}}\right)=0$. Then, the analytic cycle

$$
\left[V\left(z_{0}, \ldots, z_{s-1}, \frac{\partial f}{\partial z_{s}}, \ldots, \frac{\partial f}{\partial z_{n}}\right)\right]
$$


has the origin as a 0 -dimensional component, and $[\mathbf{0}]$ appears in this cycle with multiplicity $\mu_{\mathbf{0}}\left(f_{\mathbf{0}}\right)$. Thus, at the origin,

$$
C:=\left[V\left(\frac{\partial f}{\partial z_{s}}, \ldots, \frac{\partial f}{\partial z_{n}}\right)\right]
$$

is purely $s$-dimensional and is properly intersected by $\left[V\left(z_{0}, \ldots, z_{s-1}\right)\right]$. Let the cycle $\Gamma_{f, \mathbf{z}}^{s}$ be the sum of the components of $C$, with their multiplicities, which are not contained in $\Sigma f$, and let $\Lambda_{f, \mathbf{z}}^{s}$ be the cycle $C-\Gamma_{f, \mathbf{z}}^{s}$. The cycles $\Gamma_{f, \mathbf{z}}^{s}$ and $\Lambda_{f, \mathbf{z}}^{s}$ are, respectively, the $s$-dimensional polar cycle and $s$-dimensional Lê cycle; see [17]. It follows at once that

$$
\mu_{\mathbf{0}}\left(f_{\mathbf{0}}\right)=\left(\Gamma_{f, \mathbf{z}}^{s} \cdot V\left(z_{0}, \ldots, z_{s-1}\right)\right)_{\mathbf{0}}+\left(\Lambda_{f, \mathbf{z}}^{s} \cdot V\left(z_{0}, \ldots, z_{s-1}\right)\right)_{\mathbf{0}} .
$$

Note that $\Gamma_{f, \mathbf{z}}^{s}=0$ is equivalent to the equality of sets: $\Sigma f=V\left(\frac{\partial f}{\partial z_{s}}, \ldots, \frac{\partial f}{\partial z_{n}}\right)$.

Using our notation from the introduction, $\Lambda_{f, \mathbf{z}}^{s}=\sum_{\nu} \stackrel{\circ}{\mu}_{\nu}[\nu]$, where the sum is over the $s$-dimensional components $\nu$ of $\Sigma f$, and, by definition, $\lambda_{f, \mathbf{z}}^{s}(\mathbf{0})=$ $\left(\Lambda_{f, \mathbf{z}}^{s} \cdot V\left(z_{0}, \ldots, z_{s-1}\right)\right)_{\mathbf{0}}$. Therefore, we obtain:

Lemma 2.1. Suppose that $\operatorname{dim}_{\mathbf{0}} \Sigma\left(f_{\mathbf{0}}\right)=0$. Then,

$$
\begin{aligned}
\mu_{\mathbf{0}}\left(f_{\mathbf{0}}\right) & =\left(\Gamma_{f, \mathbf{z}}^{s} \cdot V\left(z_{0}, \ldots, z_{s-1}\right)\right)_{\mathbf{0}}+\sum_{\nu} \stackrel{\circ}{\mu}_{\nu}\left(\nu \cdot V\left(z_{0}, \ldots, z_{s-1}\right)\right)_{\mathbf{0}} \\
& =\left(\Gamma_{f, \mathbf{z}}^{s} \cdot V\left(z_{0}, \ldots, z_{s-1}\right)\right)_{\mathbf{0}}+\lambda_{f, \mathbf{z}}^{s}(\mathbf{0}),
\end{aligned}
$$

where the sum is over all s-dimensional components, $\nu$, of $\Sigma f$.

In particular, $\mu_{\mathbf{0}}\left(f_{\mathbf{0}}\right)=\lambda_{f, \mathbf{z}}^{s}(\mathbf{0})$ if and only if $\Gamma_{f, \mathbf{z}}^{s}=0$.

Note that, while $\lambda_{f, \mathbf{z}}^{s}(\mathbf{0})$ is not independent of the choice of $\mathbf{z}, \Lambda_{f, \mathbf{z}}^{s}$ is independent of the coordinate choice, and this fact is very useful. Let $\left(\hat{z}_{0}, \ldots, \hat{z}_{n}\right)$ be a set of local analytic coordinates for $\mathcal{U}$ which are close to the coordinates $\mathbf{z}$; let $\hat{f}_{\mathbf{q}}$ denote the corresponding analytic family. As $\operatorname{dim}_{\mathbf{0}} \Sigma\left(f_{\mathbf{0}}\right)=0, \operatorname{dim}_{\mathbf{0}} \Sigma\left(\hat{f}_{\mathbf{0}}\right)=$ 0 . Let $\widehat{C}:=\left[V\left(\frac{\partial f}{\partial \hat{z}_{s}}, \ldots, \frac{\partial f}{\partial \hat{z}_{n}}\right)\right]$. Then, Proposition 8.2.a of [7] implies that $\lim _{\hat{\mathbf{z}} \rightarrow \mathbf{z}} \widehat{C} \leq C$, i.e.,

$$
\lim _{\hat{\mathbf{z}} \rightarrow \mathbf{z}}\left(\Gamma_{f, \hat{\mathbf{z}}}^{s}+\Lambda_{f, \hat{\mathbf{z}}}^{s}\right) \leq\left(\Gamma_{f, \mathbf{z}}^{s}+\Lambda_{f, \mathbf{z}}^{s}\right) .
$$

As $\Lambda_{f, \mathbf{z}}^{s}$ is independent of the coordinates, we conclude that $\lim _{\hat{\mathbf{z}} \rightarrow \mathbf{z}} \Gamma_{f, \hat{\mathbf{z}}}^{s} \leq \Gamma_{f, \mathbf{z}}^{s}$.

It follows immediately that

Lemma 2.2. . If there exist coordinates $\mathbf{z}$ such that $\operatorname{dim}_{\mathbf{0}} \Sigma\left(f_{\mathbf{0}}\right)=0$ and $\Gamma_{f, \mathbf{z}}^{s}=0$, then the set of coordinates $\hat{\mathbf{z}}$ such that $\operatorname{dim}_{\mathbf{0}} \Sigma\left(\hat{f}_{\mathbf{0}}\right)=0$ and $\Gamma_{f, \hat{\mathbf{z}}}^{s}=0$ form an open dense set (i.e., the linear portion of $\hat{\mathbf{z}}$ is obtained from the standard coordinates by applying a transformation from an open dense set of $\mathrm{PGL}\left(\mathbb{C}^{n+1}\right)$ ). 
There is one more piece of preliminary notation that we need. Consider the blow-up of $\mathcal{U}$ along the Jacobian ideal, $J(f)$ of $f$, i.e., $B:=\mathrm{Bl}_{J(f)} \mathcal{U}$. This blow-up naturally sits inside $\mathcal{U} \times \mathbb{P}^{n}$. Thus, the exceptional divisor $E$ of the blow-up is a cycle in $\mathcal{U} \times \mathbb{P}^{n}$.

We now give many equivalent characterizations of $\mu$-constant families. Note that we are not presupposing below that the critical locus of $f$ is smooth at the origin.

Theorem 2.3.. Let $\mathbf{z}$ be local coordinates for $\mathcal{U}$ at the origin such that $\operatorname{dim}_{\mathbf{0}} \Sigma\left(f_{\mathbf{0}}\right)$ $=0$. Then, the following are equivalent:

1. For all $\mathbf{q} \in \Sigma f$ near the origin, $\mu_{\mathbf{0}}\left(f_{\mathbf{0}}\right)=\mu_{\mathbf{q}}\left(f_{\mathbf{q}}\right)$.

2. $\mu_{\mathbf{0}}\left(f_{\mathbf{0}}\right)=\lambda_{f}^{s}(\mathbf{0})$.

3. $f_{\mathbf{q}}$ is a simple $\mu$-constant family.

4. $\mu_{\mathbf{0}}\left(f_{\mathbf{0}}\right)=\lambda_{f, \mathbf{Z}}^{s}(\mathbf{0})$.

5. $\Gamma_{f, \mathbf{z}}^{s}=0$.

Futhermore, if $n-s \neq 2$, then 1), 2), 3), 4), and 5) above hold if and only if the local, ambient, topological-type of $V\left(f_{\mathbf{q}}\right)$ at $\mathbf{q}$ is independent of the point $\mathbf{q} \in \Sigma f$ near the origin.

In addition, the following are equivalent:

a. There exist coordinates $\mathbf{z}$ such that 1), 2), 3), 4), and 5) above hold.

b. Near the origin, $\Sigma f$ is smooth and $(\mathcal{U}-\Sigma f, \Sigma f)$ is an $a_{f}$ stratification, i.e., for all $\mathbf{p} \in \Sigma f$ near the origin, for every limiting tangent space, $\mathcal{T}_{\mathbf{p}}$, from level hypersurfaces of $f$ approaching $\mathbf{p}, T_{\mathbf{p}}(\Sigma f) \subseteq \mathcal{T}_{\mathbf{p}}$.

c. $\Sigma f$ is smooth at the origin, and over an open neighborhood of the origin, the exceptional divisor, $E$, as a set, is equal to the projectivized conormal variety to $\Sigma f$ and, hence, as cycles $E=\stackrel{\circ}{\mu}_{\Sigma f}\left[T_{\Sigma f}^{*} \mathcal{U}\right]$.

d. For generic $\hat{\mathbf{z}}, \Gamma_{f, \hat{\mathbf{z}}}^{s}=0$ near the origin.

e. $\Sigma f$ is smooth at the origin and, for all local coordinates $\hat{\mathbf{z}}$ such that $V\left(\hat{z}_{0}, \ldots\right.$, $\left.\hat{z}_{s-1}\right)$ transversely intersects $\Sigma f$ at the origin, $\hat{f}_{\mathbf{q}}$ is a simple $\mu$-constant family.

Proof. Throughout, we work in a sufficiently small neighborhood of the origin. The theorem is trivially true if $s=0$; so, we suppose that $s \geq 1$.

Suppose that 1) holds. Then, Lemma 2.1 implies that

$$
\mu_{\mathbf{0}}\left(f_{\mathbf{0}}\right)=\left(\Gamma_{f, \mathbf{z}}^{s} \cdot V\left(z_{0}, \ldots, z_{s-1}\right)\right)_{\mathbf{0}}+\sum_{\nu} \mu_{\mathbf{0}}\left(f_{\mathbf{0}}\right)\left(\nu \cdot V\left(z_{0}, \ldots, z_{s-1}\right)\right)_{\mathbf{0}} .
$$

Thus, $\Gamma_{f, \mathbf{z}}^{s}=0$, and $\sum_{\nu}\left(\nu \cdot V\left(z_{0}, \ldots, z_{s-1}\right)\right)_{\mathbf{0}}$ must equal 1, i.e., $\Sigma f$ must have a single smooth $s$-dimensional component, which is transversely intersected by 
$V\left(z_{0}, \ldots, z_{s-1}\right)$. Hence, $\lambda_{f}^{s}(\mathbf{0})=\sum_{\nu} \stackrel{\circ}{\mu}_{\nu}\left(\nu \cdot V\left(z_{0}, \ldots, z_{s-1}\right)\right)_{\mathbf{0}}$, and Lemma 2.1 now implies 2).

Suppose that 2) holds. Then, Lemma 2.1 implies that $\Gamma_{f, \mathbf{z}}^{s}=0$ and, for each $s$-dimensional component $\nu$ of $\Sigma f,\left(\nu \cdot V\left(z_{0}, \ldots, z_{s-1}\right)\right)_{\mathbf{0}}=$ mult $_{\mathbf{0}} \nu$. In order to conclude 3$)$, we have only to show that $\Sigma f$ must be smooth.

As $\Gamma_{f, \mathbf{z}}^{s}=0$, there is an equality of sets $\Sigma f=V\left(\frac{\partial f}{\partial z_{s}}, \ldots, \frac{\partial f}{\partial z_{n}}\right)$, and so every component of $\Sigma f$ must be at least $s$-dimensional. We conclude that $\Sigma f$ is purely $s$-dimensional. Now, let $\hat{\mathbf{z}}$ be a generic choice of coordinates, close to $\mathbf{z}$. As the Milnor number is upper-semicontinuous, $\mu_{\mathbf{0}}\left(f_{\mathbf{0}}\right) \geq \mu_{\mathbf{0}}\left(\hat{f}_{\mathbf{0}}\right) \geq \lambda_{f}^{s}(\mathbf{0})$. As $\mu_{\mathbf{0}}\left(f_{\mathbf{0}}\right)=$ $\lambda_{f}^{s}(\mathbf{0})$, we conclude that $\mu_{\mathbf{0}}\left(\hat{f}_{\mathbf{0}}\right)=\lambda_{f}^{s}(\mathbf{0})$. As prepolar coordinates are generic (see $[17])$, we may assume that $\hat{\mathbf{z}}$ is prepolar.

Consider now $g:=f_{\left.\right|_{V\left(\hat{z}_{0}, \ldots, \hat{z}_{s-2}\right)}}$ (where we mean that $g:=f$ if $s=1$ ). Then, as $\hat{\mathbf{z}}$ is generic, $\Sigma g=\Sigma f \cap V\left(\hat{z}_{0}, \ldots, \hat{z}_{s-2}\right)$ is 1-dimensional. By induction, Proposition 1.21 of [17] implies that the polar curve $\Gamma_{g, z_{s-1}}^{1}=\Gamma_{f, \mathbf{z}}^{s} \cdot V\left(\hat{z}_{0}, \ldots, \hat{z}_{s-1}\right)=0$. Now, Proposition 1.30 of [17] (which uses the non-splitting result, proved independently by Gabrielov [8], Lazzeri [10], and Lê [12]) implies that $\Sigma g$ is smooth. As $\hat{\mathbf{z}}$ was generic and $\Sigma f$ was purely $s$-dimensional, we conclude that $\Sigma f$ is smooth, and so 3 ) holds.

Certainly, 3) implies 1). Therefore, we have shown that 1), 2), and 3) are equivalent. By Lemma 2.1,4) and 5) are equivalent. That 3) implies 4) is immediate.

Now, suppose that 4) holds. Then, easy generalizations of any of the nonsplitting arguments of Gabrielov [8], Lazzeri [10], and Lê [12] immediately imply that, at the origin, $\Sigma f$ has a single smooth component and $V\left(z_{0}, \ldots, z_{s-1}\right)$ transversely intersects $\Sigma f$. For example, Lê's proof in our current setting is as follows. Fix a small open ball $\stackrel{\circ}{B}_{\epsilon}$ around the origin in $\mathcal{U}$, small enough so that the only critical point of $f_{\mathbf{0}}$ in $\stackrel{\circ}{B}_{\epsilon} \cap V\left(z_{0}, \ldots, z_{s-1}\right)$ is $\mathbf{0}$. For all $\mathbf{q} \in \Sigma f$ near the origin, let $\operatorname{tr}\left(f_{\mathbf{q}}\right)$ denote the trace of the Milnor monodromy of $f_{\mathbf{q}}$ on $\widetilde{H}_{n-s}\left(F_{f_{\mathbf{q}}, \mathbf{q}}\right)$. As 4) implies 5), we may follow Lê's argument in [12] to conclude that, for all $\mathbf{a}:=\left(a_{0}, \ldots, a_{s-1}\right) \in \mathbb{C}^{s}$ near the origin,

$$
\operatorname{tr}\left(f_{\mathbf{0}}\right)=\sum_{\mathbf{q} \in K} \operatorname{tr}\left(f_{\mathbf{q}}\right)
$$

where $K:=\stackrel{\circ}{B}_{\epsilon} \cap \Sigma f \cap V\left(z_{0}-a_{0}, \ldots, z_{s-1}-a_{s-1}\right)$. However, by the main theorem of [1], each of the traces above is equal to $(-1)^{n-s-1}$. Hence, the number of elements of $K$ must be precisely 1 , and so $\Sigma f$ must be smooth at $\mathbf{0}$ and transversely intersected by $V\left(z_{0}, \ldots, z_{s-1}\right)$. Combined with the definition of $\lambda_{f, \mathbf{z}}^{s}(\mathbf{0})$, this implies 3). 
Thus, 1), 2), 3), 4), and 5) are equivalent.

If 3 ) holds and $n-s \neq 2$, then the classic result of Lê and Ramanujam in [14] implies that the local, ambient, topological-type of $V\left(f_{\mathbf{q}}\right)$ at $\mathbf{q}$ is independent of the point $\mathbf{q} \in \Sigma f$ near the origin.

With no constraint on $n-s$, if the local, ambient, topological-type of $V\left(f_{\mathbf{q}}\right)$ at $\mathbf{q}$ is independent of the point $\mathbf{q} \in \Sigma f$ near the origin, then 1) holds, since the Milnor number is an invariant of this topological-type.

We need to show that a) through e) are equivalent.

Assume that a) holds. Then, 3) implies b) by Theorem 6.8 of [17] (which uses the result of Lê and Saito from [15]).

The equivalence of b) and c) is immediate, and they clearly imply d). That e) implies a) is also clear. It remains for us to show that d) implies e).

Assume d). By Lemma 2.1, a) holds and, thus, so does c). Hence, $\Sigma f$ is smooth at the origin. Let $\hat{\mathbf{z}}$ be such that $V\left(\hat{z}_{0}, \ldots, \hat{z}_{s-1}\right)$ transversely intersects $\Sigma f$ at the origin. Then, c) tells us at once that $\operatorname{dim}_{\mathbf{0}} \Sigma\left(f_{\mathbf{0}}\right)=0$ and $\Gamma_{f, \hat{\mathbf{z}}}^{s}=0$, and so, by Lemma 2.1, a) holds.

Definition 2.4.. Whenever the equivalent conditions a), b), c), d), and e) of Theorem 2.3 hold, we say that $f$ is Milnor equisingular at the origin.

The Main Theorem of this paper, Theorem 5.3, tells us that there is another topological characterization of Milnor equisingularity. First, however, we must recall some known results and prove the Swing Lemma.

\section{KnOwn Results}

We assume that the first coordinate $z_{0}$ on $\mathcal{U}$ is a generic linear form; in the terminology of [17], we need for $z_{0}$ to be "prepolar" (with respect to $f$ at the origin). This implies that $\operatorname{dim}_{0} \Sigma\left(f_{\left.\right|_{V\left(z_{0}\right)}}\right) \leq s-1$ (provided that $s \neq 0$ ), that the polar curve, $\Gamma_{f, z_{0}}^{1}$, is purely 1-dimensional at the origin (which vacuously includes the case $\Gamma_{f, z_{0}}^{1}=\emptyset$ ), and $\Gamma_{f, z_{0}}^{1}$ has no components contained in $V(f)$ (this last property is immediate in some definitions of the relative polar curve).

For convenience, we assume throughout the remainder of this paper that the neighborhood $\mathcal{U}$ is re-chosen, if necessary, so small that $\Sigma f \subseteq V(f)$, and every component of $\Sigma f$ and $\Gamma_{f, z_{0}}^{1}$ contains the origin.

There is the attaching result of Lê from [11] (see, also, [17]): 
Theorem 3.1. Up to diffeomorphism, $F_{f}$ is obtained from $\stackrel{\circ}{\mathbb{D}} \times F_{f_{0}}$ by attaching $\tau:=\left(\Gamma_{f, z_{0}}^{1} \cdot V(f)\right)_{\mathbf{0}}$ handles of index $n$.

Remark 3.2. . On the level of homology, Lê's attaching result is a type of Lefschetz hyperplane result; it says that, for all $i<n-1$, the inclusion map $F_{f_{0}}=F_{f} \cap V\left(z_{0}\right) \hookrightarrow F_{f}$ induces isomorphisms $\widetilde{H}_{i}\left(F_{f_{0}}\right) \cong \widetilde{H}_{i}\left(F_{f}\right)$, and $\widetilde{H}_{n}\left(F_{f}\right)$ and $\widetilde{H}_{n-1}\left(F_{f}\right)$ are, respectively, isomorphic to the kernel and cokernel of the boundary map

$$
\mathbb{Z}^{\tau} \cong H_{n}\left(F_{f}, F_{f_{0}}\right) \stackrel{\partial}{\longrightarrow} \widetilde{H}_{n-1}\left(F_{f_{0}}\right) .
$$

We remind the reader here of the well-known result, first proved by Teissier in [27] (in the case of an isolated singularity, but the proof works in general), that

$$
\tau=\left(\Gamma_{f, z_{0}}^{1} \cdot V(f)\right)_{\mathbf{0}}=\left(\Gamma_{f, z_{0}}^{1} \cdot V\left(\frac{\partial f}{\partial z_{0}}\right)\right)_{\mathbf{0}}+\left(\Gamma_{f, z_{0}}^{1} \cdot V\left(z_{0}\right)\right)_{\mathbf{0}} .
$$

As defined in [17], the first summand on the right above is $\lambda_{f, z_{0}}^{0}(\mathbf{0})$, the 0 dimensional Lê number, and second summand on the right above is $\gamma_{f, z_{0}}^{1}(\mathbf{0})$, the 1-dimensional polar number.

\section{Assume, throughout the remainder of this section, that $s=1$.}

In this case, $\widetilde{H}_{n-1}\left(F_{f_{0}}\right) \cong \mathbb{Z}^{\mu_{0}\left(f_{0}\right)}$, and one can certainly calculate the difference of the reduced Betti numbers of $F_{f}$ :

$$
\tilde{b}_{n}\left(F_{f}\right)-\tilde{b}_{n-1}\left(F_{f}\right)=\tau-\mu_{\mathbf{0}}\left(f_{0}\right) .
$$

Hence, a bound on one of $\tilde{b}_{n}\left(F_{f}\right)$ and $\tilde{b}_{n-1}\left(F_{f}\right)$ automatically produces a bound on the other. As a final comment, it is well-known, and easy to show that $\mu_{\mathbf{0}}\left(f_{0}\right)=\gamma_{f, z_{0}}^{1}(\mathbf{0})+\lambda_{f, z_{0}}^{1}(\mathbf{0})$.

In Proposition 3.1 of [17], the second author showed how the technique of "tilting in the Cerf diagram" or "the swing", as used by Lê and Perron in [13] could help refine the result of Theorem 3.1. Here, we state only the homological implication of Proposition 3.1 of [17].

Theorem 3.3.. The boundary map $H_{n}\left(F_{f}, F_{f_{0}}\right) \stackrel{\partial}{\longrightarrow} \widetilde{H}_{n-1}\left(F_{f_{0}}\right)$ maps a direct summand of $H_{n}\left(F_{f}, F_{f_{0}}\right)$ of rank $\gamma^{1}$ isomorphically onto a direct summand of $\widetilde{H}_{n-1}\left(F_{f_{0}}\right)$.

Thus, the rank of $\widetilde{H}_{n}\left(F_{f}\right)$ is at most $\lambda_{f, z_{0}}^{0}(\mathbf{0})$, and the rank of $\widetilde{H}_{n-1}\left(F_{f}\right)$ is at $\operatorname{most} \lambda_{f, z_{0}}^{1}(\mathbf{0})$. 
However, if one of the components $\nu$ of $\Sigma f$ is itself singular, then the above bounds on the ranks are known not to be optimal. A result of Siersma in [25], or an easy exercise using perverse sheaves (see the remark at the end of [25]), yields:

Theorem 3.4. . The rank of $\widetilde{H}_{n-1}\left(F_{f}\right)$ is at most $\sum_{\nu} \stackrel{\circ}{\mu}_{\nu}$.

In light of Theorem 3.3 and Theorem 3.4, the question is: Is it possible that $\operatorname{rank} \widetilde{H}_{n-1}\left(F_{f}\right)=\lambda_{f, z_{0}}^{1}(\mathbf{0})$ ? Of course, the answer to this question is "yes"; if $f$ is Milnor equisingular and $z_{0}$ is generic, then Theorem 3.1 tells us immediately that $\operatorname{rank} \widetilde{H}_{n-1}\left(F_{f}\right)=\lambda_{f, z_{0}}^{1}(\mathbf{0})$. Theorem 5.1 tells us that the only way for $\operatorname{rank} \widetilde{H}_{n-1}\left(F_{f}\right)$ to equal $\lambda_{f, z_{0}}^{1}(\mathbf{0})$ is for $f$ to be Milnor equisingular.

Remark 3.5. Of course, if all of the components $\nu$ of $\Sigma f$ are smooth, and $z_{0}$ is generic, then $\lambda_{f, z_{0}}^{1}(\mathbf{0})=\lambda_{f}^{1}(\mathbf{0})=\sum_{\nu} \stackrel{\circ}{\mu}_{\nu}$, and the bounds on the ranks obtained from Theorem 3.3 and Theorem 3.4 are the same. In addition, Theorem 3.4 is true with arbitrary field coefficients; this yields bounds on the possible torsion in $\widetilde{H}_{n-1}\left(F_{f}\right)$. We should also remark that the result of Siersma from [25] that we cite above can actually yield a much stronger bound if one knows certain extra topological data - specifically, one needs that the "vertical monodromies" are non-trivial.

Hence, if one of the components of $\Sigma f$ is itself singular, then $\operatorname{rank} \widetilde{H}_{n-1}\left(F_{f}\right)<$ $\lambda_{f, z_{0}}^{1}(\mathbf{0})$ by Theorem 3.4. Even in the case where all of the components of $\Sigma f$ are smooth, we could conclude that $\operatorname{rank} \widetilde{H}_{n-1}\left(F_{f}\right)<\lambda_{f, z_{0}}^{1}(\mathbf{0})$ from [25] if we knew that one of the vertical monodromies were non-trivial. However, the vertical monodromies are complicated topological data to calculate. In addition, the vertical monodromies can be trivial even when the polar curve is non-empty, i.e., when $f$ is not Milnor equisingular.

In [22], Siersma proved another closely related result, at the homotopy level. On the level of homology, what he proved was that, if $f$ is not Milnor equisingular, and $\Sigma f$ has a single smooth component, $\nu$, such that $\stackrel{\circ}{\mu}_{\nu}=1$, then $\widetilde{H}_{n-1}\left(F_{f}\right)=0$; Theorem 5.1, including the modulo $p$ statement, is a strict generalization of this homological conclusion.

In addition, we should point out that, in [4], Th. de Jong provides evidence that a result like Theorem 5.1 might be true.

Before we can prove our Main Theorem, we still need to prove the Swing Lemma.

\section{The Swing}

We prove the one-dimensional version of our Main Theorem by combining the swing technique of Theorem 3.3 and the connectivity of the vanishing cycle 
intersection diagram for isolated singularities, as was proved independently by Gabrielov in [8] and Lazzeri in [10].

In Section 3, we referred to the swing (or, tilting in the Cerf diagram), which was used by Lê and Perron in [13] and in Proposition 3.1 of [17], where the swing was used to prove Theorem 3.3. The swing has also been studied in [3], [29], [17], [31]. However, no detailed proof of the swing has appeared in the literature. As the swing is so crucial to the proof of the Main Theorem, we wish to give a careful explanation of its construction.

Suppose that $\mathcal{W}$ is an open neighborhood of the origin in $\mathbb{C}^{2}$. We will use the coordinates $x$ and $y$ on $\mathcal{W}$. For notational ease, when we restrict $x$ and $y$ to various subspaces where the domain is clear, we shall continue to write simply $x$ and $y$.

Let $C$ be a complex analytic curve in $\mathcal{W}$ such that every component of $C$ contains the origin. We assume that the origin is an isolated point in $V(x) \cap C$ and in $V(y) \cap C$, i.e., that $C$ does not have a component along the $x$ - or $y$-axis.

Below, we let $\mathbb{D}_{\epsilon}$ denote a closed disk, of radius $\epsilon$, centered at the origin, in the complex plane. We denote the interior of $\mathbb{D}_{\epsilon}$ by $\stackrel{\circ}{\mathbb{D}}_{\epsilon}$, and when we delete the origin, we shall superscript with an asterisk, i.e., $\mathbb{D}_{\epsilon}^{*}:=\mathbb{D}_{\epsilon}-\{0\}$ and $\stackrel{\circ}{\mathbb{D}}_{\epsilon}^{*}:=\stackrel{\circ}{\mathbb{D}}_{\epsilon}-\{0\}$.

We select $0<\epsilon_{2} \ll \epsilon_{1} \ll 1$ so that:

i): the "half-open" polydisk $\mathbb{D}_{\epsilon_{1}} \times{\stackrel{\circ}{\mathbb{D}} \epsilon_{2}}$ is contained in $\mathcal{W}$;

ii): $\left(\partial \mathbb{D}_{\epsilon_{1}} \times \stackrel{\circ}{\mathbb{D}}_{\epsilon_{2}}\right) \cap C=\emptyset$ (using that the origin is an isolated point in $\left.V(y) \cap C\right)$;

Note that ii) implies that $\left(\mathbb{D}_{\epsilon_{1}} \times \stackrel{\circ}{\mathbb{D}}_{\epsilon_{2}}\right) \cap C=\left(\stackrel{\circ}{\mathbb{D}}_{\epsilon_{1}} \times \stackrel{\circ}{\mathbb{D}}_{\epsilon_{2}}\right) \cap C$.

iii): $\mathbb{D}_{\epsilon_{1}} \times{\stackrel{\circ}{\mathbb{D}^{\prime}}}_{\epsilon_{2}}^{*} \stackrel{y}{\longrightarrow} \stackrel{\circ}{\mathbb{D}}_{\epsilon_{2}}^{*}$ is a proper stratified submersion, where the Whitney strata are $\partial \mathbb{D}_{\epsilon_{1}} \times{\stackrel{\circ}{\mathbb{D}^{*}}}_{\epsilon_{2}}^{*},\left(\stackrel{\circ}{\mathbb{D}}_{\epsilon_{1}} \times{\stackrel{\circ}{\mathbb{D}} \epsilon_{2}}^{*}\right)-C$, and $\left(\stackrel{\circ}{\mathbb{D}}_{\epsilon_{1}} \times{\stackrel{\circ}{\mathbb{D}} \epsilon_{2}}^{*}\right) \cap C$.

iv): $\left(\stackrel{\circ}{\mathbb{D}}_{\epsilon_{1}} \times \stackrel{\circ}{\mathbb{D}}_{\epsilon_{2}}^{*}\right) \cap C \stackrel{y}{\longrightarrow} \stackrel{\circ}{\mathbb{D}}_{\epsilon_{2}}^{*}$ is an $m$-fold covering map, where $m:=(C \cdot V(y))_{\mathbf{0}}$.

Let $D:=\left(\mathbb{D}_{\epsilon_{1}} \times \stackrel{\circ}{\mathbb{D}}_{\epsilon_{2}}\right) \cap(C \cup V(y))$. Let $\left(x_{0}, y_{0}\right) \in\left(\mathbb{D}_{\epsilon_{1}}^{*} \times{\stackrel{\circ}{\mathbb{D}} \epsilon_{2}}^{*}\right)-D$. Let $\sigma:[0,1] \rightarrow\left\{x_{0}\right\} \times \stackrel{\circ}{\mathbb{D}}_{\epsilon_{2}}$ be a smooth, simple path such that $\sigma(0)=\left(x_{0}, y_{0}\right)$, $\sigma(1)=:\left(x_{0}, y_{1}\right) \in C$, and $\sigma([0,1)) \subseteq\left(\left\{x_{0}\right\} \times \stackrel{\circ}{\mathbb{D}}_{\epsilon_{2}}\right)-D$.

Let $S$ be the image of $\sigma$; as $\sigma$ is simple, $S$ is homeomorphic to [0,1]. Let $\sigma_{0}:=y \circ \sigma$ and let $S_{0}$ be the image of $\sigma_{0}$. Thus, $S_{0}$ is homeomorphic to $[0,1]$ and is contained in ${\stackrel{\circ}{\mathbb{D}_{2}}}_{\epsilon_{2}}^{*}$. 
Lemma 4.1..(The Swing) There exists a continuous function $H:[0,1] \times[0,1] \rightarrow$ $\mathbb{D}_{\epsilon_{1}} \times S_{0}$ with the following properties:

a) $H(t, 0)=\sigma(t)$, for all $t \in[0,1]$;

b) $H(t, 1) \in \mathbb{D}_{\epsilon_{1}} \times\left\{y_{0}\right\}$, for all $t \in[0,1]$;

c) $H(0, u)=\left(x_{0}, y_{0}\right)$, for all $u \in[0,1]$;

d) if $H(t, u) \in D$, then $t=1$;

e) $H(1, u) \in C$, for all $u \in[0,1]$;

f) the path $\eta$ given by $\eta(u):=H(1, u)$ is a homeomorphism onto its image.

Thus, $H$ is a homotopy from $\sigma$ to the path $\gamma$ given by $\gamma(t):=H(t, 1) \in \mathbb{D}_{\epsilon_{1}} \times$ $\left\{y_{0}\right\}$, such that $\left(x_{0}, y_{0}\right)$ is "fixed" and the point $\left(x_{0}, y_{1}\right)=H(1,0)$ "swings up to the point" $H(1,1)$ by "sliding along" $C$, while the remainder of $\sigma$ does not hit $D$ as it "swings up" to $\gamma$.

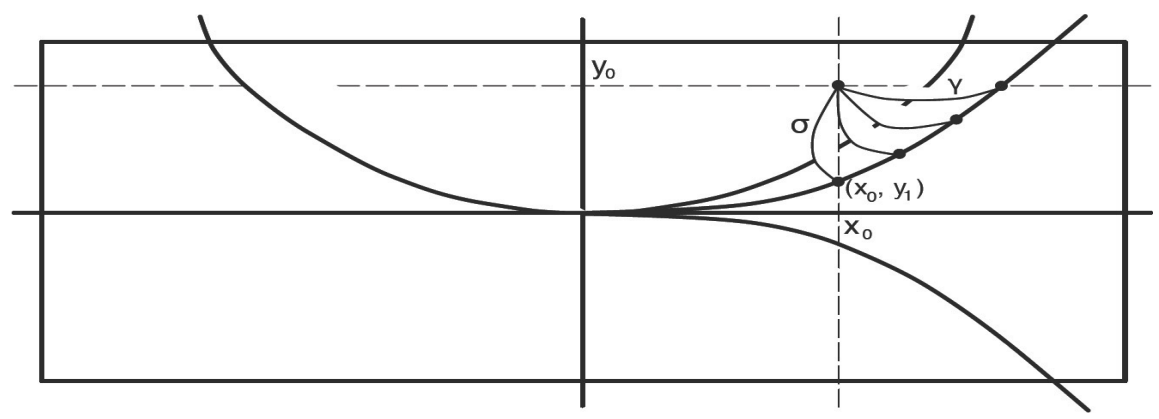

Proof. The proper stratified submersion $\mathbb{D}_{\epsilon_{1}} \times \stackrel{\circ}{\mathbb{D}_{\epsilon_{2}}^{*}} \stackrel{y}{\longrightarrow}{\stackrel{\circ}{\mathbb{D}_{2}}}_{\epsilon_{2}}^{*}$ is a locally trivial fibration, where the local trivialization respects the strata. The restriction of this fibration $\mathbb{D}_{\epsilon_{1}} \times S_{0} \stackrel{y}{\longrightarrow} S_{0}$ is a locally trivial fibration over a contractible space and, hence, is equivalent to the trivial fibration.

Therefore, there exists a homeomorphism

$$
\Psi:\left(\mathbb{D}_{\epsilon_{1}} \times S_{0},\left(\mathbb{D}_{\epsilon_{1}} \times S_{0}\right) \cap C\right) \rightarrow\left(\mathbb{D}_{\epsilon_{1}} \times\left\{y_{0}\right\},\left(\mathbb{D}_{\epsilon_{1}} \times\left\{y_{0}\right\}\right) \cap C\right) \times[0,1]
$$

such that the projection of $\Psi\left(x, \sigma_{0}(t)\right)$ onto the $[0,1]$ factor is simply $t$, and such that $\Psi\left(x, y_{0}\right)=\left(\left(x, y_{0}\right), 0\right)$. It follows that there is a path $\alpha:[0,1] \rightarrow \mathbb{D}_{\epsilon_{1}}$ such that $\Psi(\sigma(t))=\left(\left(\alpha(t), y_{0}\right), t\right)$, for all $t \in[0,1]$. Define $H:[0,1] \times[0,1] \rightarrow \mathbb{D}_{\epsilon_{1}} \times S_{0}$ by

$$
H(t, u):=\Psi^{-1}\left(\left(\alpha(t), y_{0}\right),(1-u) t\right) .
$$

All of the given properties of $H$ are now trivial to verify.

Remark 4.2. By Property c) of Lemma 4.1, the map $H$ yields a corresponding map $H^{T}$ whose domain is a triangle instead of a square. One pictures the image of $H$, or of $H^{T}$, as a "gluing in" of this triangle into $\mathbb{D}_{\epsilon_{1}} \times S_{0}$ in such a way 
that one edge of the triangle is glued diffeomorphically to $S$, and another edge is glued diffeomorphically onto the image of $\eta$. The third edge of the triangle is glued onto the image of $\gamma$, but not necessarily in a one-to-one fashion.

\section{The Main Theorem}

In this section, we will prove the Main Theorem. We must first describe the machinery that goes into this part of the proof.

We will first prove a 1-dimensional version of the Main Theorem. Assume for now that $\operatorname{dim}_{0} \Sigma f=1$. As the value of $\lambda_{f, z_{0}}^{1}(\mathbf{0})$ is minimal for generic $z_{0}$, we lose no generality if we assume that our linear form $z_{0}$ is chosen more generically than simply being prepolar. We choose $z_{0}$ so generically that, in addition to being prepolar, the discriminant, $D$, of the map $\left(z_{0}, f\right)$ and the corresponding Cerf diagram, $C$, have the usual properties - as given, for instance, in [13], [29], and [31]. We will describe the needed properties below.

Let $\widetilde{\Psi}:=\left(z_{0}, f\right):(\mathcal{U}, \mathbf{0}) \rightarrow\left(\mathbb{C}^{2}, \mathbf{0}\right)$. We use the coordinates $(u, v)$ on $\mathbb{C}^{2}$. The critical locus $\Sigma \widetilde{\Psi}$ of $\widetilde{\Psi}$ is the union of $\Sigma f$ and $\Gamma_{f, z_{0}}^{1}$. The discriminant $D:=$ $\widetilde{\Psi}(\Sigma \widetilde{\Psi})$ consists of the $u$-axis together with the Cerf diagram $C:=\overline{D-V(v)}$. We assume that $z_{0}$ is generic enough so that the polar curve is reduced and that, in a neighborhood of the origin, $\widetilde{\Psi}_{\left.\right|_{\Gamma_{f, z_{0}}^{1}}}$ is one-to-one.

We choose real numbers $\epsilon, \delta$, and $\omega$ so that $0<\omega \ll \delta \ll \epsilon \ll 1$. Let $B_{\epsilon} \subseteq \mathbb{C}^{n}$ be a closed ball, centered at the origin, of radius $\epsilon$. Let $\stackrel{\circ}{\mathbb{D}}_{\delta}$ and $\stackrel{\circ}{\mathbb{D}}_{\omega}$ be open disks in $\mathbb{C}$, centered at 0 , of radii $\delta$ and $\omega$, respectively.

One considers the map from $\left(\stackrel{\circ}{\mathbb{D}}_{\delta} \times B_{\epsilon}\right) \cap f^{-1}\left(\stackrel{\circ}{\mathbb{D}}_{\omega}\right)$ onto $\stackrel{\circ}{\mathbb{D}}_{\delta} \times \stackrel{\circ}{\mathbb{D}}_{\omega}$ given by the restriction of $\widetilde{\Psi}$; we let $\Psi$ denote this restriction. As $B_{\epsilon}$ is a closed ball, the map $\Psi$ is certainly proper, but the domain has an interior stratum, and a stratum coming from the boundary of $B_{\epsilon}$. However, for generic $z_{0}$, all of the stratified critical points lie on $\Gamma_{f, z_{0}}^{1} \cup \Sigma f$, i.e., above $D$.

We continue to write simply $D$ and $C$, in place of $D \cap\left(\stackrel{\circ}{\mathbb{D}}_{\delta} \times \stackrel{\circ}{\mathbb{D}}_{\omega}\right)$ and $C \cap$ $\left(\stackrel{\circ}{\mathbb{D}} \delta \times \stackrel{\circ}{\mathbb{D}}_{\omega}\right)$. As $\Psi$ is a proper stratified submersion above $\stackrel{\circ}{\mathbb{D}}_{\delta} \times \stackrel{\circ}{\mathbb{D}}_{\omega}-D$, and as $\Psi_{\Gamma_{f, z_{0}}^{1}}$ is one-to-one, many homotopy arguments in $\left(\stackrel{\circ}{\mathbb{D}} \delta_{\delta} \times B_{\epsilon}\right) \cap f^{-1}\left(\stackrel{\circ}{\mathbb{D}}_{\omega}\right)$ can be obtained from lifting constructions in $\stackrel{\circ}{\mathbb{D}}_{\delta} \times \stackrel{\circ}{\mathbb{D}}_{\omega}$. This is the point of considering the discriminant and Cerf diagram.

Let $v_{0} \in \stackrel{\circ}{\mathbb{D}}_{\omega}-\{\mathbf{0}\}$. By construction, up to diffeomorphism, $\Psi^{-1}\left(\stackrel{\circ}{\mathbb{D}} \delta_{\delta} \times\left\{v_{0}\right\}\right)$ is $F_{f}$ and $\Psi^{-1}\left(\left(0, v_{0}\right)\right)$ is $F_{f_{0}}$. In fact, for all $u_{0}$, where $\left|u_{0}\right| \ll\left|v_{0}\right|, \Psi^{-1}\left(\left(u_{0}, v_{0}\right)\right)$ is diffeomorphic to $F_{f_{0}}$; we fix such a non-zero $u_{0}$, and let $\mathbf{a}:=\left(u_{0}, v_{0}\right)$. 
We wish to pick a distinguished basis for the vanishing cycles of $f_{0}$ at the origin, as in I.1 of [2] (see, also, [5]). We do this by selecting paths in $\left\{u_{0}\right\} \times \stackrel{\circ}{\mathbb{D}} \omega_{\omega}$ which originate at $\mathbf{a}$. We must be careful in how we do this.

First, fix a path $p_{0}$ from a to $\left(u_{0}, 0\right)$. Select paths $q_{1}, \ldots, q_{\gamma^{1}}$ from a to each of the points in $\left(\left\{u_{0}\right\} \times \stackrel{\circ}{\mathbb{D}} \omega_{\omega}\right) \cap C=:\left\{y_{1}, \ldots, y_{\gamma^{1}}\right\}$. The paths $p_{0}, q_{1}, \ldots, q_{\gamma^{1}}$ should not intersect each other and should intersect the set $\left\{\left(u_{0}, 0\right), y_{1}, \ldots, y_{\gamma^{1}}\right\}$ only at the endpoints of the paths. Moreover, when at the point a, the paths $p_{0}, q_{1}, \ldots, q_{\gamma^{1}}$ should be in clockwise order. Let $r_{0}$ be a clockwise loop very close to $p_{0}$, from a around $\left(u_{0}, 0\right)$.

As we are not assuming that $f$ had an isolated line singularity, we must perturb $f_{\left.\right|_{V\left(z_{0}-u_{0}\right)}}$ slightly to have $\left(u_{0}, 0\right)$ split into $\lambda^{1}$ points, $x_{1}, \ldots, x_{\lambda^{1}}$ inside the loop $r_{0}$; each of these points corresponds to an $A_{1}$ singularity in the domain. We select paths $p_{1}, \ldots, p_{\lambda^{1}}$ from a to each of the points $x_{1}, \ldots, x_{\lambda^{1}}$, and paths $q_{1}, \ldots, q_{\gamma^{1}}$ from a to each of the points in $\left(\left\{u_{0}\right\} \times \mathbb{D}_{\omega} \omega\right) \cap C=:\left\{y_{1}, \ldots, y_{\gamma^{1}}\right\}$. We may do this in such a way that the paths $p_{1}, \ldots, p_{\lambda^{1}}, q_{1}, \ldots, q_{\gamma^{1}}$ are in clockwise order.
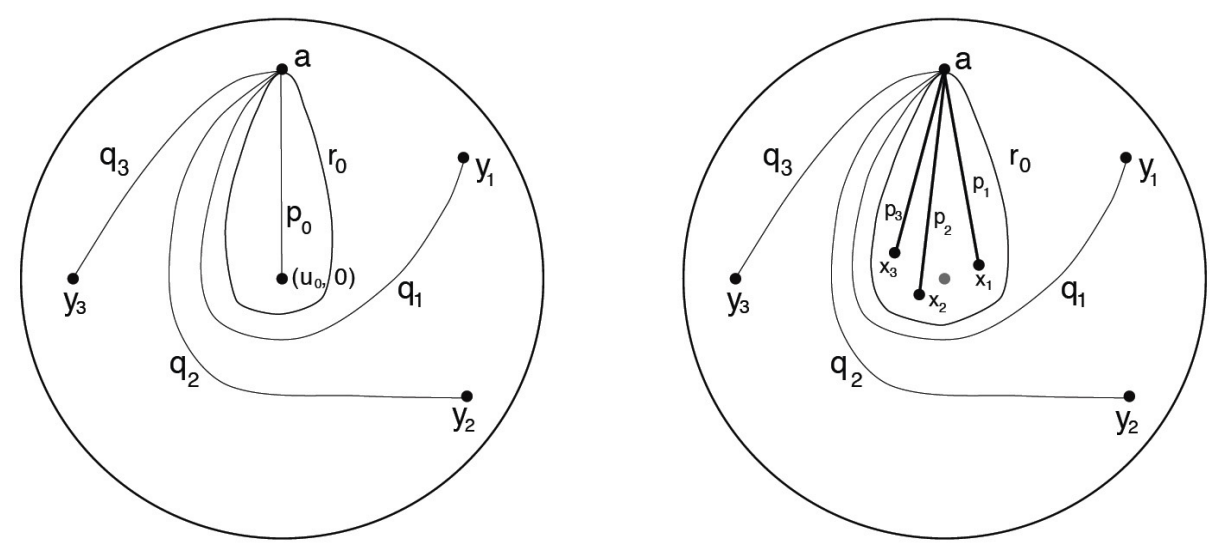

The lifts of these paths via the perturbed $f_{\left.\right|_{V\left(z_{0}-u_{0}\right)}}$ yield representatives of elements of $H_{n+1}\left(B_{\epsilon}, F_{f_{0}}\right)$, whose boundaries in $\widetilde{H}_{n}\left(F_{f_{0}}\right)$ form a distinguished basis $\Delta_{1}^{\prime}, \ldots, \Delta_{\lambda^{1}}^{\prime}, \Delta_{1}, \ldots, \Delta_{\gamma^{1}}$.

By using the swing (Lemma 4.1), the paths $q_{1}, \ldots, q_{\gamma^{1}}$ are taken to new paths $\hat{q}_{1}, \ldots, \hat{q}_{\gamma^{1}}$ in $\stackrel{\circ}{\mathbb{D}}_{\delta} \times\left\{v_{0}\right\}$. Each $\hat{q}_{i}$ path represents a relative homology class in $H_{n}\left(F_{f}, F_{f_{0}}\right)$ whose boundary in $\widetilde{H}_{n-1}\left(F_{f_{0}}\right)$ is precisely $\Delta_{i}$. We remark that Theorem 3.3 follows from this.

We can now prove: 
Theorem 5.1.. Suppose that $\operatorname{dim}_{0} \Sigma f=1$ and $\operatorname{dim}_{0} \Sigma f_{0}=0$. Then, the following are equivalent:

a) $f_{\mathbf{q}}$ is a simple $\mu$-constant family, i.e., $f$ has a smooth critical locus which defines a family of isolated singularities with constant Milnor number $\mu_{f_{0}}$;

b) $\operatorname{rank} \widetilde{H}_{n-1}\left(F_{f}\right)=\lambda_{f, z_{0}}^{1}(\mathbf{0})$;

c) there exists a prime $p$ such that $\operatorname{dim} \widetilde{H}_{n-1}\left(F_{f} ; \mathbb{Z} / p \mathbb{Z}\right)=\lambda_{f, z_{0}}^{1}(\mathbf{0})$.

Thus, if $f$ is not Milnor equisingular, and $\mathfrak{K}$ is a prime field (i.e., $\mathbb{Q}$ or $\mathbb{Z} / p \mathbb{Z}$ ), then $\operatorname{dim} \widetilde{H}_{n-1}\left(F_{f} ; \mathfrak{K}\right)<\lambda_{f, z_{0}}^{1}(\mathbf{0})$, and so $\operatorname{dim} \widetilde{H}_{n}\left(F_{f} ; \mathfrak{K}\right)<\lambda_{f, z_{0}}^{0}(\mathbf{0})$.

In particular, if $f$ is not Milnor equisingular, and $\lambda_{f, z_{0}}^{1}(\mathbf{0})=1$, then $\widetilde{H}_{n-1}\left(F_{f} ; \mathbb{Z}\right)$ $=0$ and $\widetilde{H}_{n}\left(F_{f} ; \mathbb{Z}\right)$ is free Abelian of rank $\lambda_{f, z_{0}}^{0}(\mathbf{0})-1$.

Proof. That a) implies b) and c) is well-known; it follows at once from Theorem 3.1. Assume then that $f_{\mathbf{q}}$ is not a simple $\mu$-constant family. We will prove that $\operatorname{rank} \widetilde{H}_{n-1}\left(F_{f}\right)<\lambda_{f, z_{0}}^{1}(\mathbf{0})$, and then indicate why the same proof applies with $\mathbb{Z} / p \mathbb{Z}$ coefficients.

By Theorem 2.3, $\Gamma_{f, z_{0}}^{1} \neq \emptyset$, and so $C \neq \emptyset$. We want to construct just one new path in $\left\{u_{0}\right\} \times \stackrel{\circ}{\mathbb{D}} \omega_{\omega}$, one which originates at $\mathbf{a}$, ends at a point of $C$, and misses all of the other points of $D$; we want this path to swing up to a path in $\mathbb{D}_{\delta} \times\left\{v_{0}\right\}$, and represent a relative homology class in $H_{n}\left(F_{f}, F_{f_{0}}\right)$ whose boundary is not in the span of $\Delta_{1}, \ldots, \Delta_{\gamma^{1}}$.

By the connectivity of the vanishing cycle intersection diagram ([8], [10]), one of the $\Delta_{j}^{\prime}$ must have a non-zero intersection pairing with one of the $\Delta_{i}$, i.e., there exist $i_{0}$ and $j_{0}$ such that $\left\langle\Delta_{i_{0}}, \Delta_{j_{0}}^{\prime}\right\rangle \neq 0$.

By fixing the path $p_{j_{0}}$ and all the $q_{i}$ paths, but reselecting the other $p_{j}$, for $j \neq j_{0}$, we may assume that $j_{0}=1$, i.e., that $\left\langle\Delta_{i_{0}}, \Delta_{1}^{\prime}\right\rangle \neq 0$.

We now follow Chapter 3.3 of [5]. Associated to each path $p_{j}, 1 \leqslant j \leqslant \lambda^{1}$, is a (partial) monodromy automorphism $T_{j}^{\prime}: \widetilde{H}_{n-1}\left(F_{f_{0}}\right) \rightarrow \widetilde{H}_{n-1}\left(F_{f_{0}}\right)$, induced by taking a clockwise loop $r_{j}$ very close to $p_{j}$, from a around $x_{j}$. Let $T^{\prime}:=T_{1}^{\prime} \ldots T_{\lambda^{1}}^{\prime}$, where composition is written in the order of [5]. We claim that $T^{\prime}\left(\Delta_{i_{0}}\right)$ is in the image of $\delta: H_{n}\left(F_{f}, F_{f_{0}}\right) \rightarrow \widetilde{H}_{n-1}\left(F_{f_{0}}\right)$, but is not in $\operatorname{Span}\left\{\Delta_{1}, \ldots, \Delta_{\gamma^{1}}\right\}$.

The composition $r$ of the loops $r_{1}, \ldots, r_{\lambda^{1}}$ is homotopy-equivalent, in $\left\{u_{0}\right\} \times$ $\stackrel{\circ}{\mathbb{D}} \omega_{\omega}-\left\{\left\{x_{1}, \ldots, x_{\lambda^{1}}\right\} \cup C\right\}$, to the loop $r_{0}$ (from our discussion before the theorem). By combining (concatenating) the loop $r_{0}$ and the path $q_{i_{0}}$, we obtain a path in 
$\left\{u_{0}\right\} \times \stackrel{\circ}{\mathbb{D}} \omega_{\omega}$ which is homotopy-equivalent to a simple path which swings up to a corresponding path in $\stackrel{\circ}{\mathbb{D}} \delta_{\delta} \times\left\{v_{0}\right\}$. Thus, $T^{\prime}\left(\Delta_{i_{0}}\right)$ is in the image of $\delta$.

Now, by the Corollaries to the Picard-Lefschetz Theorem in [2], p. 26, or as in [5], Formula 3.11,

$$
T^{\prime}\left(\Delta_{i_{0}}\right)=\Delta_{i_{0}}-(-1)^{\frac{n(n-1)}{2}}\left\langle\Delta_{i_{0}}, \Delta_{1}^{\prime}\right\rangle \Delta_{1}^{\prime}+\beta_{2} \Delta_{2}^{\prime}+\cdots+\beta_{\lambda^{1}} \Delta_{\lambda^{1}}^{\prime},
$$

for some integers $\beta_{2}, \ldots, \beta_{\lambda^{1}}$. As the $\Delta_{1}^{\prime}, \ldots, \Delta_{\lambda^{1}}^{\prime}, \Delta_{1}, \ldots, \Delta_{\gamma^{1}}$ form a basis, and as $\left\langle\Delta_{i_{0}}, \Delta_{1}^{\prime}\right\rangle \neq 0, T^{\prime}\left(\Delta_{i_{0}}\right)$ is not in $\operatorname{Span}\left\{\Delta_{1}, \ldots, \Delta_{\gamma^{1}}\right\}$.

This finishes the proof over the integers. Over $\mathbb{Z} / p \mathbb{Z}$, the proof is identical, since the intersection diagram is also connected modulo $p$; see [8].

Remark 5.2. One must be careful in the proof above; it is tempting to try to use simply $T_{1}^{\prime}\left(\Delta_{i_{0}}\right)$ in place of $T^{\prime}\left(\Delta_{i_{0}}\right)$. The problem with this is that $T_{1}^{\prime}\left(\Delta_{i_{0}}\right)$ is not represented by a path in $\left\{u_{0}\right\} \times \stackrel{\circ}{\mathbb{D}} \omega_{\omega}-\left\{\left(u_{0}, 0\right)\right\}$ and, thus, there is no guaranteed swing isotopy to a corresponding path in $\stackrel{\circ}{\mathbb{D}} \delta_{\delta} \times\left\{v_{0}\right\}$.

In fact, we could have avoided the explicit construction of $T^{\prime}\left(\Delta_{i_{0}}\right)$ completely, though we find the construction intuitive and geometrically interesting. By naturality (of the monodromy automorphism on the vanishing cycle functor), the map $\delta: H_{n}\left(F_{f}, F_{f_{0}}\right) \rightarrow \widetilde{H}_{n-1}\left(F_{f_{0}}\right)$ commutes with the respective monodromy actions. Thus, the image of $\delta, \operatorname{im} \delta$, is invariant under the monodromy action. Now, the swing and the construction of the distinguished basis for $\widetilde{H}_{n-1}\left(F_{f_{0}}\right)$ tell us that we can write $\widetilde{H}_{n-1}\left(F_{f_{0}}\right)$ as a direct sum $A \oplus B$, where $A$ and $B$ are generated by distinguished basis elements, $\operatorname{rank} A=\gamma^{1}$, and $A \subseteq \operatorname{im} \delta$. However, the connectivity of the intersection matrix for $f_{0}$ implies that the only monodromy-invariant submodules of $\widetilde{H}_{n-1}\left(F_{f_{0}}\right)$, which are generated by distinguished basis elements, are the zero-module and all of $\widetilde{H}_{n-1}\left(F_{f_{0}}\right)$ (see [2], Theorem 3.5). Thus, if $\gamma^{1} \neq 0$ (i.e., if we do not have a simple $\mu$-constant family), then the image of $\delta$ must properly contain $A$. Theorem 5.1 follows.

We should also remark that using $\mathfrak{K}:=\mathbb{Z} / p \mathbb{Z}$ coefficients in Theorem 5.1, even when $\lambda_{f, z_{0}}^{1}(\mathbf{0}) \geq 2$, yields non-trivial information. By the Universal Coefficient Theorem and because $\widetilde{H}_{n-2}\left(F_{f} ; \mathbb{Z}\right)=0$, we find that $\widetilde{H}_{n-1}\left(F_{f} ; \mathfrak{K}\right) \cong$ $\widetilde{H}_{n-1}\left(F_{f} ; \mathbb{Z}\right) \otimes \mathfrak{K}$. The dimension of this $\mathfrak{K}$-vector space is equal to the rank of $\widetilde{H}_{n-1}\left(F_{f} ; \mathbb{Z}\right)$ plus the number of cyclic $p$-torsion direct summands of $\widetilde{H}_{n-1}\left(F_{f} ; \mathbb{Z}\right)$, and so, if $f$ is not Milnor equisingular, then $\left(\lambda_{f, z_{0}}^{1}(\mathbf{0})-1\right)$ is an upper-bound on this sum.

Finally, as we mentioned earlier, while our result is on the homology level, one may also investigate this situation on the homotopy level, as in [22] (in the case 
$\lambda_{f, z_{0}}^{1}(\mathbf{0})=1$ ) and in [30] (in the general $s=1$ case). The homology statement in Theorem 5.1 follows from these homotopy results.

We can now prove our Main Theorem. We return to the general case where $s:=\operatorname{dim}_{0} \Sigma f$ is arbitrary. Fix a set of coordinates $\left(z_{0}, \ldots, z_{n}\right)$, and consider the corresponding family $f_{\mathbf{q}}$.

Theorem 5.3. (Main Theorem). Suppose that $\operatorname{dim}_{\mathbf{0}} \Sigma\left(f_{\mathbf{0}}\right)=0$. Let $\mathfrak{K}$ be a prime field.

Then, $\operatorname{dim} \widetilde{H}_{n-s}\left(F_{f} ; \mathfrak{K}\right)=\lambda_{f, \mathbf{z}}^{s}(\mathbf{0})$ if and only if $f_{\mathbf{q}}$ is a simple $\mu$-constant family.

Proof. If $f_{\mathbf{q}}$ is a simple $\mu$-constant family, then it is well-known that $\operatorname{dim} \widetilde{H}_{n-s}$ $\left(F_{f} ; \mathfrak{K}\right)=\lambda_{f, \mathbf{z}}^{s}(\mathbf{0})$; this follows from an inductive application of [11], using that $\Gamma_{f, \mathbf{z}}^{s}=0$ (as we saw in Theorem 2.3).

Suppose that $\tilde{b}_{n-s}:=\operatorname{dim} \widetilde{H}_{n-s}\left(F_{f} ; \mathfrak{K}\right)=\lambda_{f, \mathbf{z}}^{s}(\mathbf{0})$. As we saw in the Introduction, $\tilde{b}_{n-s} \leq \lambda_{f}^{s}(\mathbf{0}) \leq \lambda_{f, \mathbf{z}}^{s}(\mathbf{0})$. Thus, we must have that $\lambda_{f, \mathbf{z}}^{s}(\mathbf{0})=\lambda_{f}^{s}(\mathbf{0})$. Let $\hat{\mathbf{z}}$ be a generic choice of coordinates at the origin, consider the codimension $s-1$ linear slice $N:=V\left(\hat{z}_{0}, \ldots, \hat{z}_{s-2}\right)$ through the origin.

Then, $f_{\left.\right|_{N}}$ has a 1-dimensional critical locus and, by an iterated application of Theorem 3.1, we obtain that $\widetilde{H}_{n-s}\left(F_{f} ; \mathfrak{K}\right)$ is isomorphic to $\widetilde{H}_{(n-s+1)-1}\left(F_{f_{\left.\right|_{N}}} ; \mathfrak{K}\right)$. Now, by Proposition 1.21 of $[17], \lambda_{f, \hat{\mathbf{z}}}^{s}(\mathbf{0})=\lambda_{f_{\left.\right|_{N}}, \hat{z}_{s-1}}^{1}(\mathbf{0})$. Combining these last two sentences with Theorem 5.3, we conclude that $f_{\left.\right|_{N}}$ is Milnor equisingular; in particular, the polar curve, $\Gamma_{f_{\left.\right|_{N}}, \hat{z}_{s-1}}$ is zero (or, as a set, is empty). By Proposition 1.21 of [17], this implies that $\Gamma_{f, \hat{\mathbf{z}}}^{s}=0$. Now, by d) of Theorem 2.3,f is Milnor equisingular. Therefore, $\Sigma f$ is smooth at the origin and, since $\lambda_{f, \mathbf{z}}^{s}(\mathbf{0})=$ $\lambda_{f}^{s}(\mathbf{0}), V\left(z_{0}, \ldots, z_{s-1}\right)$ must transversely intersect $\Sigma f$ at the origin. The desired conclusion now follows from e) of Theorem 2.3.

In the following corollary and in Proposition 5.6, we will use terminology and techniques involving perverse sheaves and the complex of vanishing cycles. We refer the reader to [6] and [18], Appendix B.

We wish to see that Theorem 5.3 puts restrictions on the types of perverse sheaves that one may obtain as shifted vanishing cycles $\phi_{f}[-1] \mathbb{Z}_{\mathcal{U}}^{\bullet}[n+1]$ of the shifted constant sheaf on affine space. We will restrict this perverse sheaf to its support. Below, we refer to the constant sheaf on $\nu$ of rank $\stackrel{\circ}{\mu}_{\nu}$, shifted by some integer $j$; we write $\left(\mathbb{Z}^{\stackrel{\circ}{\mu}}\right)_{\nu}^{\bullet}[j]$ for this sheaf. The isomorphisms and direct sums that we write below are in the Abelian category of perverse sheaves. 
Corollary 5.4.. Suppose that the critical locus of $f$ is s-dimensional, where $s \geqslant 1$ is arbitrary, and that every s-dimensional component, $\nu$, of $\Sigma f$ is smooth.

Then, $\bigoplus_{\nu}\left(\mathbb{Z}^{\stackrel{\rho}{\mu}_{\nu}}\right)_{\nu}^{\bullet}[s]$ is a direct summand of $\left(\phi_{f}[-1] \mathbb{Z}_{\mathcal{U}}^{\bullet}[n+1]\right)_{\left.\right|_{\Sigma f}}$ if and only if $f$ is Milnor equisingular. Moreover, when these equivalent conditions hold, $\Sigma f$ is smooth and $\left(\phi_{f}[-1] \mathbb{Z}_{\mathcal{U}}^{\bullet}[n+1]\right)_{\left.\right|_{\Sigma f}} \cong\left(\mathbb{Z}^{\mu_{\Sigma f}}\right)_{\Sigma f}^{\bullet}[s]$.

In addition, this conclusion holds with $\mathbb{Z}$ replaced by any prime field.

Proof. We will give the proof with $\mathbb{Z}$ coefficients. The proof modulo $p$ is identical.

If $f$ is Milnor equisingular, then, by b) of Theorem 2.3, $V(f)$ has an $a_{f}$ strat-

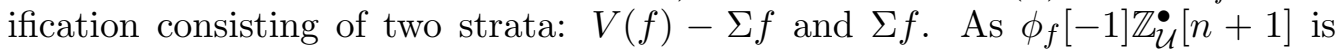
constructible with respect to any $a_{f}$ stratification, it follows that

$$
\left(\phi_{f}[-1] \mathbb{Z}_{\mathcal{U}}^{\bullet}[n+1]\right)_{\left.\right|_{\Sigma f}} \cong\left(\mathbb{Z}^{\stackrel{\circ}{\mu f}}\right)_{\Sigma f}^{\bullet}[s] .
$$

If $\bigoplus_{\nu}\left(\mathbb{Z}^{\stackrel{\rho}{\mu}_{\nu}}\right)_{\nu}^{\bullet}[s]$ is a direct summand of $\left(\phi_{f}[-1] \mathbb{Z}_{\mathcal{U}}^{\bullet}[n+1]\right)_{\mid \Sigma f}$, then $\operatorname{rank} \widetilde{H}_{n-s}\left(F_{f}\right)$ is at least $\sum_{\nu} \stackrel{\circ}{\mu}_{\nu}$, which equals $\lambda_{f}^{s}(\mathbf{0})$, as each $\nu$ is smooth. Now, Theorem 5.3 tells us that $f$ must be Milnor equisingular.

Not surprisingly, if one places more restrictions on the critical locus of $f$, one can obtain restrictions on the cohomology of the Milnor fiber that are sharper than what we obtain in Theorem 5.3. For example, in [20] and [32], Némethi and Zaharia consider the case where the critical locus is a 2-dimensional complete intersection with an isolated singularity and, for each 2-dimensional component $\nu$ of $\Sigma f, \stackrel{\circ}{\mu}_{\nu}=1$; in addition, they place special assumptions on the types of critical points of $f$ that one can have at non-generic points of $\Sigma f-\{\mathbf{0}\}$.

We will prove a result related to those in in [20] and [32]. Our hypotheses on the critical locus are substantially weaker than those in in [20] and [32], and our conclusion is correspondingly not as strong. First, we need a new definition.

Definition 5.5.. A point $\mathbf{p} \in \Sigma f$ is a Milnor equisingular point of $f$ if and only if there exists an open neighborhood $\mathcal{W}$ of $\mathbf{p}$ in $\mathcal{U}$ such that $f_{\left.\right|_{\mathcal{W}}}$ is Milnor equisingular.

Proposition 5.6.. Suppose that $s=\operatorname{dim}_{0} \Sigma f \geq 2$, and that for all $s$-dimensional components $\nu$ of $\Sigma f, \stackrel{\circ}{\mu}_{\nu}=1$. Suppose that there exists an open neighborhood $\mathcal{W}$ of the origin in $\mathcal{U}$ and an analytic function $g:(\mathcal{W} \cap \Sigma f, \mathbf{0}) \rightarrow(\mathbb{C}, 0)$ such that

(1) $\mathcal{W} \cap \Sigma f-\{\mathbf{0}\}$ is smooth,

(2) for all $\mathbf{p} \in \mathcal{W} \cap \Sigma f-V(g)$, $\mathbf{p}$ is a Milnor equisingular point of $f$, and

(3) for all $\mathbf{p} \in \mathcal{W} \cap V(g)-\{\mathbf{0}\}$, $\mathbf{p}$ is not a Milnor equisingular point of $f$. 
Then, $\widetilde{H}^{n-s}\left(F_{f}\right)=0$.

Proof. Throughout the proof, we shall work in a sufficiently small neighborhood $\mathcal{W}$, in which all of the hypotheses of the proposition are satisfied. We shall suppress any further reference to $\mathcal{W}$. Note that Item 3 above excludes the possibility that $g$ vanishes identically on a component of $\Sigma f$ containing the origin.

Let $\mathbf{P}^{\bullet}$ be the perverse sheaf on $\Sigma f$ given by restricting the shifted vanishing cycles to their support, i.e.,

$$
\mathbf{P}^{\bullet}:=\left(\phi_{f}[-1] \mathbb{Z}_{\mathcal{U}}[n+1]\right)_{\left.\right|_{\Sigma f}} .
$$

Let $\Sigma^{*}:=\Sigma f-\{\mathbf{0}\}$. Let $\hat{\imath}$ be the open inclusion of $\Sigma f-V(g)$ into $\Sigma^{*}$, let $m$ be the open inclusion of $\Sigma^{*}$ into $\Sigma f$, and let $i:=m \circ \hat{\imath}$.

The cosupport condition for perverse sheaves, applied at the origin, immediately implies that

$$
\widetilde{H}^{n-s}\left(F_{f}\right) \cong H^{-s}\left(\mathbf{P}^{\bullet}\right)_{\mathbf{0}} \cong \mathbb{H}^{-s}\left(\Sigma^{*} ; \mathbf{P}_{\left.\right|_{\Sigma^{*}}}\right)
$$

Using the $E^{2}$ spectral sequence for hypercohomology and that $\mathbf{P}_{\left.\right|_{\Sigma^{*}}}$ is perverse, we conclude that $\mathbb{H}^{-s}\left(\Sigma^{*} ; \mathbf{P}_{\left.\right|_{\Sigma^{*}}}\right) \cong H^{0}\left(\Sigma^{*} ; \mathbf{H}^{-s}\left(\mathbf{P}_{\left.\right|_{\Sigma^{*}}}\right)\right)$; see, for instance, [5], Proposition 5.2.20. By Theorem 5.3 or $[22], \mathbf{H}^{-s}\left(\mathbf{P}_{\left.\right|_{\Sigma^{*}}}\right)$ is a local system in degree $-s$, extended by zero over $V(g)-\{\mathbf{0}\}$, i.e., if $\mathbf{Q}^{\bullet}$ is the complex of sheaves which has $\mathbf{H}^{-s}\left(\mathbf{P}_{\left.\right|_{\Sigma^{*}}}\right)$ in degree $-s$, and zero in all other degress, then $\mathbf{Q}^{\bullet} \cong$ $\hat{\imath}_{!} \hat{\imath}^{!}\left(\mathbf{P}_{\left.\right|_{\Sigma^{*}}}\right) \cong\left(i_{i} i^{i} \mathbf{P}^{\bullet}\right)_{\left.\right|_{\Sigma^{*}}}$. Therefore,

$$
H^{0}\left(\Sigma^{*} ; \mathbf{H}^{-s}\left(\mathbf{P}_{\left.\right|_{\Sigma^{*}}}\right)\right) \cong \mathbb{H}^{-s}\left(\Sigma^{*} ; \mathbf{Q}^{\bullet}\right) \cong \mathbb{H}^{-s}\left(\Sigma^{*} ;\left(i_{i} i \mathbf{P}^{\bullet}\right)_{\left.\right|_{\Sigma^{*}}}\right)
$$

Now, the extension by zero of a perverse sheaf on the complement of a hypersurface defined by a single function is perverse; see, for instance, [18], Appendix B, p. 210.

Once again using the cosupport condition for perverse sheaves, we find that

$$
\mathbb{H}^{-s}\left(\Sigma^{*} ;\left(i_{i} i \mathbf{P}^{\bullet}\right)_{\Sigma_{\Sigma^{*}}} \cong H^{-s}\left(i_{i} i \mathbf{P}^{\bullet}\right)_{\mathbf{0}}=0\right.
$$

Combining all of the above isomorphisms yields $\widetilde{H}^{n-s}\left(F_{f}\right)=0$.

Remark 5.7.. Note that, if $\Sigma f$ is smooth at the origin, then Proposition 5.6 follows at once from Theorem 5.3, which - since we have assumed that $\stackrel{\circ}{\mu}_{\nu}=1-$ follows quickly from the main theorem of [22]. 


\section{Comments, Questions, And Counterexamples}

One might hope that a stronger result than Theorem 5.3, or Theorem 5.1, is true.

For instance, given that Theorem 5.1 and Theorem 3.4 are true, it is natural to ask the following:

Question 6.1.. If we are not in the trivial case, is the rank of $\widetilde{H}_{n-1}\left(F_{f}\right)$ strictly less than $\sum_{\nu} \stackrel{\circ}{\mu}_{\nu}$ ?

The answer to the above question is "no". One can find examples of this in the literature (see, for instance, [24], [19], [26]); perhaps the easiest is the following:

Example 6.2. . Let $f:=\left(y^{2}-x^{3}\right)^{2}+w^{2}$. Then, $\Sigma f$ has a single component $\nu:=V\left(w, y^{2}-x^{3}\right)$, and one easily checks that $\stackrel{\circ}{\mu}_{\nu}=1$. However, as $f$ is the suspension of $\left(y^{2}-x^{3}\right)^{2}$, the Sebastiani-Thom Theorem (here, we need the version proved by Oka in [21]) implies

$$
\widetilde{H}_{1}\left(F_{f}\right) \cong \widetilde{H}_{0}\left(F_{\left(y^{2}-x^{3}\right)^{2}}\right) \cong \mathbb{Z}
$$

and

$$
\widetilde{H}_{2}\left(F_{f}\right) \cong \widetilde{H}_{1}\left(F_{\left(y^{2}-x^{3}\right)^{2}}\right) \cong \mathbb{Z}^{4} .
$$

Moreover, by suspending $f$ again, one may produce an example in which $f$ itself has a single irreducible component at the origin.

We wish to see that this example also shows that the assumption on the smoothness of the $s$-dimensional components of $\Sigma f$ in Corollary 5.4 is necessary. This will require a short discussion about perverse sheaves.

Let $\mathfrak{K}$ be a prime field. We claim that, for the current example, the shifted constant sheaf $\mathfrak{K}_{\Sigma f}^{\bullet}[1]$ is a direct summand of $\left(\phi_{f}[-1] \mathfrak{K}_{\mathcal{U}}^{\bullet}[n+1]\right)_{\Sigma f}$ in the Abelian category of germs of perverse sheaves at the origin in $\Sigma f$.

The critical locus in our example has a single component at the origin. Hence, the germ of $\Sigma f$ at the origin is topologically a complex line (a real 2-disk). The category of germs, at the origin, of perverse sheaves on a line is equivalent to a category in which the objects are diagrams $A \stackrel{\alpha}{\longrightarrow} B \stackrel{\beta}{\longrightarrow} A$, where $A$ and $B$ are finite-dimensional $\mathfrak{K}$-vector spaces, and id $-\beta \circ \alpha$ is an isomorphism. A morphism between two diagrams $A_{1} \stackrel{\alpha_{1}}{\longrightarrow} B_{1} \stackrel{\beta_{1}}{\longrightarrow} A_{1}$ and $A_{2} \stackrel{\alpha_{2}}{\longrightarrow} B_{2} \stackrel{\beta_{2}}{\longrightarrow} A_{2}$ consists of 
linear transformations $f: A_{1} \rightarrow A_{2}$ and $g: B_{1} \rightarrow B_{2}$ such that

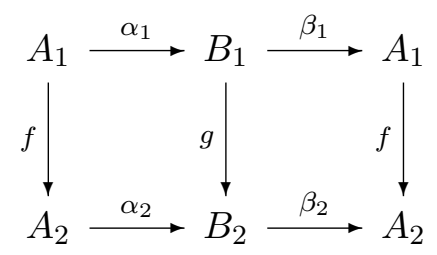

commutes. See [16]. We refer to these diagrams as MacPherson-Vilonen diagrams or, simply, $M V$-diagrams. The Verdier dual of the perverse sheaf represented by $A \stackrel{\alpha}{\longrightarrow} B \stackrel{\beta}{\longrightarrow} A$ has as its MV-diagram the dual (in the linear algebra sense) diagram $A^{*} \stackrel{\beta^{*}}{\longrightarrow} B^{*} \stackrel{\alpha^{*}}{\longrightarrow} A^{*}$.

Suppose now that $\mathbf{P}^{\bullet}$ is a perverse sheaf on the germ of a complex line at the origin. While we do not wish to explain how one obtains the vector space $B$ or the maps $\alpha$ and $\beta$ of the corresponding MV-diagram, we need to explain enough to reach the conclusion that we desire. In the MV-diagram corresponding to $\mathbf{P}^{\bullet}$, the vector space $A$ is isomorphic to $H^{-1}\left(\mathbf{P}^{\bullet}\right)_{x}$ at any $x \neq 0$ which is close to 0 . In addition, the map $\alpha$ in the MV-diagram is such that $\operatorname{ker} \alpha \cong H^{-1}\left(\mathbf{P}^{\bullet}\right)_{0}$ and coker $\alpha \cong H^{0}\left(\mathbf{P}^{\bullet}\right)_{0}$. Finally, the shifted constant sheaf of dimension $d$ corresponds to the MV-diagram $\mathfrak{K}^{d} \rightarrow 0 \rightarrow \mathfrak{K}^{d}$.

Now, consider $\mathbf{P}^{\bullet}:=\left(\phi_{f}[-1] \mathfrak{K}_{\mathcal{U}}^{\bullet}[n+1]\right)_{\left.\right|_{\Sigma f}}$ and its MV-diagram $A \stackrel{\alpha}{\longrightarrow} B \stackrel{\beta}{\longrightarrow} A$. In our current example, the dimension of $A$ is $\stackrel{\circ}{\mu}_{\nu}=1$, and $\operatorname{dim} H^{-1}\left(\mathbf{P}^{\bullet}\right)_{0}$ is equal to the dimension of $\widetilde{H}^{1}\left(F_{f} ; \mathfrak{K}\right)$, which is 1 . As ker $\alpha \cong H^{-1}\left(\mathbf{P}^{\bullet}\right)_{0}, \alpha$ must be the zero map. As $\alpha=0, B \cong$ coker $\alpha \cong H^{0}\left(\mathbf{P}^{\bullet}\right)_{0} \cong \widetilde{H}^{2}\left(F_{f} ; \mathfrak{K}\right) \cong \mathfrak{K}^{4}$.

In addition, the vanishing cycles are self-dual; that is, the Verdier dual of $\mathbf{P}^{\bullet}$ is isomorphic to itself. It follows that $\beta$ must also be the zero map. It is now a trivial exercise to show that the MV-diagram of $\left(\phi_{f}[-1] \mathfrak{K}_{\mathcal{U}}^{\bullet}[n+1]\right)_{\left.\right|_{\Sigma f}}$ is the direct sum of $\mathfrak{K} \rightarrow 0 \rightarrow \mathfrak{K}$ and $0 \rightarrow \mathfrak{K}^{4} \rightarrow 0$, i.e., the shifted constant sheaf $\mathfrak{K}_{\Sigma f}^{\bullet}[1]$ is a direct summand of $\left(\phi_{f}[-1] \mathfrak{K}_{\mathcal{U}}^{\bullet}[n+1]\right)_{\mid \Sigma f}$.

The reader should understand part of the significance of this example. While a perverse sheaf is a topological device which does not distinguish between the germ of a complex line and the germ of an irreducible curve, the vanishing cycles can "remember" that they come from a function whose critical locus is not smooth and, thus, unlike the case in Corollary 5.4, the shifted constant sheaf is "allowed" to be a direct summand.

Now, let $\alpha$ be the number of irreducible components of $\Sigma f$.

Question 6.3. If we are not in the trivial case, is the rank of $\widetilde{H}_{n-1}\left(F_{f}\right)$ strictly less than $\lambda^{1}-\alpha$ ? 
Again, there are examples in the literature (see, for instance, [23]) which demonstrate that the answer to this question is "no". One simple example is:

Example 6.4. . The function $f=x^{2} y^{2}+w^{2}$ has a critical locus consisting of two lines, $\lambda^{1}=2$, but - using the Sebastiani-Thom Theorem again - we find that $\widetilde{H}_{1}\left(F_{f}\right) \cong \mathbb{Z}$.

However, a result such as that asked about in Question 6.3, but where $\alpha$ is replaced by a quantity involving the number of components of $\Gamma_{f, z_{0}}^{1}$, or numbers of various types of components in the Cerf diagram, seems more likely. Moreover, if we put more conditions on the intersection diagram for the vanishing cycles of $f_{0}$, we could certainly obtain sharper bounds than we do in the Main Theorem. Or, if we know more topological data, such as the vertical monodromies, as in [25], we could obtain better bounds. However, other than Theorem 5.1 and Theorem 5.3, we know of no nice, effectively calculable, bound which holds in all cases.

Finally, Corollary 5.4 and Example 6.2 lead us to ask:

Question 6.5. Which perverse sheaves can be obtained as the vanishing cycles of the constant sheaf on affine space? In particular, if we fix an irreducible curve $C \subseteq \mathcal{U}$ containing the origin, can we list precisely those MV-diagrams which can be obtained from the vanishing cycles of $f: \mathcal{U} \rightarrow \mathbb{C}$, where $C=\Sigma f$ ?

Unlike our previous questions, we do not know the answer to Question 6.5.

\section{REFERENCES}

[1] A'Campo, N. Le nombre de Lefschetz d'une monodromie. Proc. Kon. Ned. Akad. Wet., Series A, 76:113-118, 1973.

[2] Arnold, V. I., Gusein-Zade, S. M., Varchenko, A. N. Singularities of Differentiable Maps II, Monodromy and Asymptotics of Integrals. Birkhäuser, 1988.

[3] Caubel, C. Sur la topologie d'une famille de pinceaux de germes d'hypersurfaces complexes. $\mathrm{PhD}$ thesis, Université Toulouse III, 1998.

[4] de Jong, Th. Some classes of line singularities. Math. Zeitschrift, 198:493-517, 1998.

[5] Dimca, A. Singularities and Topology of Hypersurfaces. Universitext. Springer-Verlag, 1992.

[6] Dimca, A. Sheaves in Topology. Universitext. Springer-Verlag, 2004.

[7] Fulton, W. Intersection Theory, volume 2 of Ergeb. Math. Springer-Verlag, 1984.

[8] Gabrielov, A. M. Bifurcations, Dynkin Diagrams, and Modality of Isolated Singularities. Funk. Anal. Pril., 8 (2):7-12, 1974.

[9] Kato, M. and Matsumoto, Y. On the connectivity of the Milnor fibre of a holomorphic function at a critical point. Proc. of $19^{773}$ Tokyo manifolds conf., pages 131-136, 1973.

[10] Lazzeri, F. Some Remarks on the Picard-Lefschetz Monodromy. Quelques journées singulières. Centre de Math. de l'Ecole Polytechnique, Paris, 1974.

[11] Lê, D. T. Calcul du Nombre de Cycles Evanouissants d'une Hypersurface Complexe. Ann. Inst. Fourier, Grenoble, 23:261-270, 1973.

[12] Lê, D. T. Une application d'un théorème d'A'Campo a l'equisingularité. Indag. Math., 35:403-409, 1973. 
[13] Lê, D. T. and Perron, B. Sur la fibre de Milnor d'une singularité isolée en dimension complexe trois. C. R. Acad. Sci. Pairs Sér. A, 289:115-118, 1979.

[14] Lê, D. T. and Ramanujam, C. P. The Invariance of Milnor's Number implies the Invariance of the Topological Type. Amer. Journ. Math., 98:67-78, 1976.

[15] Lê, D. T. and Saito, K. La constance du nombre de Milnor donne des bonnes stratifications. C.R. Acad. Sci., 277:793-795, 1973.

[16] MacPherson, R. and Vilonen, K. Elementary construction of perverse sheaves. Invent. Math., 84:403-435, 1986.

[17] Massey, D. Lê Cycles and Hypersurface Singularities, volume 1615 of Lecture Notes in Math. Springer-Verlag, 1995.

[18] Massey, D. Numerical Control over Complex Analytic Singularities, volume 778 of Memoirs of the AMS. AMS, 2003.

[19] Némethi, A. The Milnor fibre and the zeta function of the singularities of the type $f=$ $P(h, g)$. Compositio Math., 79:63-97, 1991.

[20] Némethi, A. Hypersurface Singularities with 2-dimensional Critical Locus. J. London Math. Soc. (2), 59:922-938, 1999.

[21] Oka, M. On the homotopy type of hypersurfaces defined by weighted homogeneous polynomials. Topology, 12:19-32, 1973.

[22] Siersma, D. Isolated line singularities. Proc. Symp. Pure Math., 35, part 2, Arcata Singularities Conf.:485-496, 1983.

[23] Siersma, D. Singularities with critical locus a 1-dimensional complete intersection and transversal type $A_{1}$. Top. and its Appl., 27:51-73, 1987.

[24] Siersma, D. Hypersurfaces with singular locus a plane curve and transversal type $A_{1} . B a$ nach Center Publications, Warsaw, 40:397-410, 1988.

[25] Siersma, D. Variation mappings on singularities with a 1-dimensional critical locus. Topology, 30:445-469, 1991.

[26] Siersma, D. The Vanishing Topology of Non-Isolated Singularities. In New Developments in Singularity Theory, pages 447-472. Kluwer Academic Publishers, 2001. D. Siersma, C.T.C. Wall, and V. Zakalyukin (eds.).

[27] Teissier, B. Cycles évanescents, sections planes et conditions de Whitney. Astérisque, 78:285-362, 1973.

[28] Teissier, B. Résolution simultanée et cycles évanescents. In Sém. sur les singularités des surfaces, Proc. 1976-77, Springer Lecture Notes in Math., 777, pages 82-146. SpringerVerlag, 1980. M. Demazure, H. Pinkham, and B. Teissier (eds.).

[29] Tibăr, M. Bouquet Decomposition of the Milnor Fiber. Topology, 35:227-241, 1996.

[30] Tibăr, M. The Vanishing Neighborhood of Non-isolated Singularities. to appear in Israel J. Math, 2006.

[31] Vannier, J. P. Familles à paramètre de fonctions holomorphes à ensemble singulier de dimension zéro ou un. $\mathrm{PhD}$ thesis, Dijon, 1987.

[32] Zaharia, A. Topological Properties of certain Singularities with Critical Locus a 2dimensional Complete Intersection. Topology and its Appl., 60:153-171, 1994.

Lê Dũng Tráng

ICTP, Mathematics Section

Strada Costiera 11

I-34014 Trieste (Italy)

E-mail: ledt@ictp.it
David B. Massey

Department of Mathematics

567 Lake Hall

Northeastern University

Boston, MA 02115 USA

E-mail: dmassey@neu.edu 